\title{
Physics in One Dimension
}

\author{
A subject of broad relevance throughout the field of the physics \\ of condensed matter
}

\section{J.A. Krumhansl, Cornell}

After two years of planning by a number of physicists who recognized an identity of interests among their generally disjointed subfields of condensed matter, a meeting sponsored by EPS, on Physics in One Dimension was held in Fribourg, Switzerland on 24-29 August with as main organizers J. Bernasconi and R. Orbach. The programme emphasized Nonlinear Phenomena, Disorder and Localization, Lattice Dynamics, Magnetic Chain Systems, Conducting Polymers, and 1-D Conductors.

It was apparent from the beginning that the planning committee's hopes were being met: the participants shared many common interests and a high level of attendance was maintained throughout the week. Although the phenomena which were discussed occur generally for all condensed matter systems, they are strongly enhanced in highly anisotropic or nearly one-dimensional materials - it is this feature which brought together many participants who recognized the similarity of concepts which have arisen in vastly different experimental contexts.

\section{Overview of the Programme}

It was Mattis and Lieb's book Mathematical Physics in One Dimension which, in the early 1960 's, set the stage for both the theoretical and experimental developments, so that it was appropriate that Mattis should give a key-note address on how to reduce practically any problem to one dimension. The subsequent invited talks thoroughly reviewed the present status of theory and experiment in the broad field of one-dimensional physics, and they also revealed many open and controversial aspects.

A particularly interesting feature of onedimensional materials is their instability, or at least incipient instability, toward both dynamic and structural changes. Corresponding problems were discussed in a number of lectures on one-dimensional electronic and ionic conductors, as well as in extensive mathematical investigations.

One of the most active areas of research in one-dimensional physics today is that associated with conducting polymers. Polyacetylene was the focus of both an afternoon session and an evening roundtable, with attention being paid to such aspects as doping effects, conductivity and spin resonance measurements, the theory of solitonic states in dimerized systems, and the nature of the insulator-to-metal transition.

Reviews were given on the quantum aspects of spin chains, on the general features to be expected in scattering studies of spin dynamics, and on the experimental work that has been performed on a variety of one-dimensional magnetic materials.

Extensive discussions were held on the very interesting effects of disorder in nearly one-dimensional systems. The respective sessions included lectures on the spectral properties of disordered chains, on excitation dynamics in random one-dimensional systems, and on random exchange antiferromagnetic spin chains. Much excitement was generated by the current theoretical and experimental work on strong quantum effects (i.e. localization) in disordered quasi-one-dimensional systems. Mention should also be made of the very recent experimental work on molecular chain compounds made possible by high resolution lasers.

Finally, there were the many discussions of the mathematical physics related to nonlinear soliton-like excitations. Surveys were concerned with the inverse spectral theory of solitonic states, with quantum inverse scattering theory, and with classical and quantum statistics of solitons. They were complemented by molecular dynamics investigations and by a discussion of the nucleation approach to non-linear multistable systems.

\section{Stimulus to Measurement and Mate- rials Science}

It is apparent that the needs for structural analysis and the study of dynamic phenomena in nearly one-dimensional materials have presented great challenges to experimentalists. Indeed, the extension of $\mathrm{X}$-ray and neutron scattering methods to determine structure and dynamics in charge and spin density wave systems, can be considered a remarkable achievement. Similarly, the successes of spin resonance, ultrasonics, and magnetic susceptibility measurements on small samples are both exciting and quantitatively informative.

Great challenges have been presented also to materials scientists: physicists, and metallurgists have been led to create a fascinating new wave of magnetic systems, organic conducting salts, polymers, and ferroelectrics.

\section{Properties and Relevance of One- Dimensional Models}

The ability to solve problems in one dimension can lend considerable insight into many systems, and there exist certain characteristics of mathematical models and physical theories (e.g. perturbation series and their diagrammatics) which are peculiar to one dimension. Whereas one might suspect that these simplifications become unrealistic for three-dimensional systems, even if they are highly anisotropic, many important phenomena seem to be amplified by compression into one dimension, among them fluctuations, nonlinearity, long relaxation phenomena, and the appearance of metastable states. It is worth noting that most of the recent mathematical progress in solving problems of nonlinear dynamics (e.g. solitons) has been made on one-dimensional systems and that some of the significant features have been identified in anisotropic systems of higher dimensionality.

Another theoretical development, both fascinating and of considerable conceptual utility, is the exploitation of the fact that ddimensional quantum systems at zero temperature can be mapped onto $d+1$-dimensional classical systems at finite temperature. This association has not only extended the number of methods available for treating problems, but has served to bring statistical mechanics and quantum field theory much closer together. At the Conference the relationship was emphasized particularly in connection with anisotropic magnetic systems.

\section{Numerical Work}

The importance of numerical work was a recurrent theme throughout the conference. Computational treatments of onedimensional systems may provide a more convenient realization of physical models than is possible in higher dimensions either because of limited computer capacity or because boundary effects are less significant in one-dimensional simulations. Examples of the utility of the computational approach were the calculation of quantum statistical mechanical properties of finite magnetic chains and the dynamics of the coupled electron-ion system in polyacetylene, where it was possible to trace the connection between solitons and electronic excitations. There have also been substantial studies of finite temperature dynamics in a variety of lattice systems. It is apparent that both the pictorial information that one can obtain by using modern computers and the unique interaction between the physicist and the computer are fertile additions 
to theoretical physics in general and to the solution of one-dimensional problems in particular.

\section{New Chemical States}

Physicists have long been interested in broken symmetry and strong-coupling in physical systems. Both of these features appear in the description of solitons in polyacetylene, where symmetry is broken in the dimerization pattern by a domain wall, and the microscopics of such a soliton emerges itself from a complete strong-coupling solution (in Hartree approximation) to the coupled electronphonon field problem. Indeed, generalizing from this example, one can seriously consider the possibility of many new "chemical states" of matter, with various kinds of conformational symmetry modifications coupled strongly to extended modifications in the electronic configuration.

\section{Structure and Transport}

Also discussed at the Conference were some apparently contradictory properties of one-dimensional materials. $\mathrm{Hg}_{3-x} \mathrm{AsF}_{6}$, for example, shows condensed mass density waves, yet there is no electronic transition directly associated with this condensation. Superconductivity occurs in $\mathrm{TMTSF}_{2}$ $\mathrm{PF}_{6}$, yet does not seem to be related to observed structural phase changes. Very nonlinear electrical transport is observed in $\mathrm{NbSe}_{3}$ while the system simultaneously remains conducting throughout a series of structural phase changes. These are obviously not simple Peierls systems; it seems certain that much more remains to be learned about interacting electrons and electron-ion systems. Perhaps we are really beginning to see some of the distinctly different behaviours predicted for

\section{0-31 July, 1981. \\ NATO ADVANCED STUDY INSTITUTE \\ University of Antwerp (U.I.A.), Belgium \\ ELECTRON CORRELATIONS IN SOLIDS (with emphasis on metals)}

Spin and charge density waves, density functional formalism, transport properties of many electron systems, exchange and correlation, local fields, effective interactions in metals, Wigner condensation, inelastic electron scattering in metals and plasmon dispersion experiments, plasmons in inhomogeneous many electron systems, collective excitations in molecules, atoms, solids.

Scientific Committee:

Prof. J.T. Devreese, Chairman

Dr. F. Brosens

Department of Physics, University of Antwerpen

Dr. P. Van Camp Universiteitsplein 1

B - 2610 WILRIJK

Tel. : (31) 282528

different ranges of coupling constants ("gology") in the Luttinger model.

\section{Effects of Disorder}

One-dimensional systems certainly bring out important features due to randomness. These effects may be mild, characteristic, or strong. In mild effects, only average consequences of the statistical properties appear. There are, however, many examples where the specific distribution of the random feature places a characteristic signature on the phenomena in question, for example, relaxation rates and excitation transfer in one-dimensional systems. Finally, there are strong and fundamental consequences of randomness, such as the localization of quantum states in long but not completely one-dimensional systems, experimental evidence for which was presented at the Conference. This is one of the most interesting current research areas in physics.

\section{Conclusion}

The topics discussed can be seen as covering many current themes in condensed matter physics, e.g. equilibrium proper- ties (statistical mechanics, thermodynamics); charge and spin density waves; transitional states (such as clusters, nuclei of new phases, domain walls, etc.); and the effects of disorder. Each of these aspects is of interest in its own right, but their interplay seems most pronounced in one-dimensional systems.

The topics thus span almost the entire spectrum of problems in condensed matter physics, possibly with the exception of critical phenomena associated with phase transitions (which occur only in systems of higher dimensionality). Because of this conceptual breadth and the challenges presented to experimentalists and materials scientists, the subject of one-dimensional physics will certainly be an active one for some time to come.

The Conference Proceedings which result (Springer Series in Solid-State Sciences, 1981) should be an excellent guide to this important area of physics, chemistry, and applied mathematics. Abstracts of the papers presented at the Conference have been published in the Europhysics Conference Abstracts Series, Volume 4C.

\section{New Individual Ordinary Members of EPS}

\author{
Category 4A \\ J. Hugill, Abingdon, UK \\ E.G. Schröter, Freiburg, D \\ Category $4 \mathrm{C}$ \\ Eötvős Lorand Physical Society: \\ D.L. Beke, Debrecen \\ P. Richter, Budapest \\ Finnish Physical Society: \\ M. Krusius, Turku \\ E. Laine, Turku \\ M. Punkkinen, Turku \\ French Physical Society: \\ J. André, Marseille \\ G. Bonneaud, Stanford, USA \\ A. Lacaze, Grenoble \\ D. Launois, Orsay \\ J. Monin, Saint Etienne \\ L. Monnerie, Paris \\ D. Thoulouze, Grenoble
}

Forms for applying to become a member of the European Physical Society are available from the Secretariat in Geneva. Conference organizers and heads of department are invited to state their requirements for bulk quantities for distribution to potential candidates. Full membership of a European National Society gives automatic qualification.

\author{
German Physical Society: \\ I. Broser, Berlin \\ G. Fritzsch, Frankfurt/M \\ R. Gerhard, Darmstadt \\ U. Kopacz, Braunschweig \\ The Institute of Physics \\ M. Laugier, Coventry \\ T.S. Moss, Malvern \\ D.H. Roberts, Wembley \\ Italian Physical Society: \\ W.P. Trower, Blacksburg, USA \\ The Netherlands' Physical Society: \\ A. Miedema, Eindhoven
}

\author{
Norwegian Physical Society: \\ B. Berre, Aas \\ K.J. Mork, Trondheim \\ I. Röeggen, Tromsö \\ Spanish Royal Society of Physics \\ and Chemistry: \\ J.A. Valles-Abarca, Santander \\ Swedish Physical Society \\ S.P. Hörnfeldt, Täby \\ T. Lavold, Järvälla \\ M. Skalberg, Kopparberg \\ L. Söderholm, Täby \\ Swiss Physical Society: \\ H.P. Eichenberger, Zurich
}

